\title{
Buckling of Euler Columns with a Continuous Elastic Restraint via Homotopy Analysis Method
}

\author{
Aytekin Eryılmaz, ${ }^{1}$ M. Tarık Atay, ${ }^{2}$ Safa B. Coşkun, ${ }^{3}$ and Musa Başbük ${ }^{1}$ \\ ${ }^{1}$ Department of Mathematics, Nevşehir University, 50300 Nevşehir, Turkey \\ ${ }^{2}$ Department of Mathematics, Niğde University, 51100 Niğde, Turkey \\ ${ }^{3}$ Department of Civil Engineering, Kocaeli University, 41380 Kocaeli, Turkey
}

Correspondence should be addressed to Aytekin Eryılmaz; eryilmazaytekin@gmail.com

Received 12 October 2012; Revised 10 December 2012; Accepted 10 December 2012

Academic Editor: Fazlollah Soleymani

Copyright (C) 2013 Aytekin Eryllmaz et al. This is an open access article distributed under the Creative Commons Attribution License, which permits unrestricted use, distribution, and reproduction in any medium, provided the original work is properly cited.

Homotopy Analysis Method (HAM) is applied to find the critical buckling load of the Euler columns with continuous elastic restraints. HAM has been successfully applied to many linear and nonlinear, ordinary and partial, differential equations, integral equations, and difference equations. In this study, we presented the application of HAM to the critical buckling loads for Euler columns with five different support cases continuous elastic restraints. The results are compared with the analytic solutions.

\section{Introduction}

The research area of buckling of nonuniform columns has been one of the important topics of extensive studies based on the reality that is closely related to the fields of structural, mechanical, and aeronautical engineering. Determination of practical load carrying capacity of a structural member requires a detailed stability analysis in theoretical and computational manner. Columns are one of the most used basic structural elements, and there are extensive studies related to the elastic stability of columns with different properties in shape and of material and to their static and dynamic behaviors. Many types of structures and structural members can be defined as a uniform and/or non-uniform column in a simplified state with different end conditions for buckling analysis. On the other hand, it is difficult to determine the exact analytical solutions for these buckling problems of various column types with arbitrary distributions of flexural stiffness and various end conditions. Conducting research on buckling of columns has become the center point of study for many researchers, and studying this subject becomes more and more systematic during the last decades. As a starting point of this line of research topic, Euler's early study of buckling of columns under their own weight [1] can be counted. Afterwards, Greenhill [2] made remarkable contributions to this field. In this field of study, generally, the closed form solutions are extremely hard to establish. However, solutions for simple cases are found by Dinnik [3], Karman and Biot [4], Timoshenko and Gere [5], and others. Wang et al. [6] established exact solutions for buckling of structural members including various cases of columns, beams, arches, rings, plates, and shells. In addition to this line of research, the columns with variable cross-section, some exact solutions are given in terms of logarithmic and trigonometric functions by Bleich [7], in terms of Bessel functions by Dinnik [8] and in terms of Lommel functions by Elishakoff and Pellegrini [911]. Exact solution by series representation for buckling load for variable cross section columns with variable axial forces was established by Eisenberger [12]. Exact buckling solutions for several special types of tapered columns with simple boundary conditions were given by Gere and Carter [13] with Bessel functions. Moreover, solutions for a problem of the buckling of elastic columns with step varying thicknesses are given by Arbabi and Li [14]. Siginer [15] conducted research on the stability of a column whose flexural rigidity has a continuous linear variation along the column. Furthermore, the 


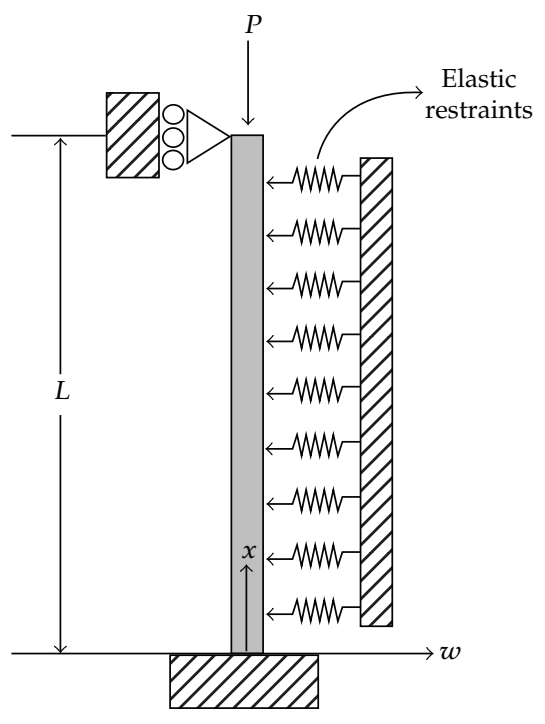

Figure 1: Column with continuous elastic restraints.

exact analytical solutions of a one-step bar and multistep bar with varying cross section under the action of concentrated and variably distributed axial loads were obtained by Li et al. [16-18]. Sampaio and Hundhausen [19] gave the solution for the problem of buckling behavior of inclined beam column using energy method. They formulated the exact solution using generalized hypergeometric functions. Moreover, the researchers who studied the mechanical behavior of beams/columns can be given as Keller [20], Tadjbakhsh and Keller [21], and Taylor [22]. A number of researches on this topic have been made by Atay and Coşkun to investigate the elastic stability of a homogenous and nonhomogenous Euler beam by using variational iteration method and homotopy perturbation method [23-29]. The problem of stability analysis of non-uniform rectangular beams, such as lateral torsional buckling of rectangular beams, was solved by using homotopy perturbation method by Pinarbasi [30]. By transforming the governing equation with varying coefficients to linear algebraic equations and also by using various end boundary conditions, critical buckling loads of beams with arbitrarily axial inhomogeneity are solved by Huang and Luo [31]. Recently, Yuan and Wang [32] used a new differential quadrature based iterative numerical integration method to solve postbuckling differential equations of extensible beam columns with six different cases.

Liao [33] introduced Homotopy Analysis Method (HAM) to obtain series solutions of various linear and nonlinear problems. HAM is an efficient method that presents us acceptable analytical results with convenient convergence [33]. In opposition to the perturbation techniques, this approach is independent of any small parameters, and HAM provides us with a simple procedure to obtain the convergence of series of solutions so that one can obtain accurate enough approximations by auxiliary convergence controller parameter $\hbar$. Liao solved many linear and nonlinear problems by HAM. In his book, especially, he points out the basic ideas of the HAM [33-36]. Recently, this technique has successfully been applied to several nonlinear problems such as the viscous flows of non-Newtonian fluids [37, 38], nonlinear heat transfer [39], nonlinear Fredholm integral equations [40], the KdV-type equations [41], differential difference equations [42], time-dependent Emden-Fowler type equations [43], Laplace equation with Dirichlet and Neumann conditions [44], and multipantograph equations [45].

In this study we apply Homotopy Analysis Method (HAM) to find the critical buckling load of elastic columns with continuous restraints. This problem has been solved by different approaches such as Variational Iteration Method (VIM), but Ham has some advantages such as being based on a generalized concept of the homotopy in topology; the HAM has the following advantages. The HAM is always valid no matter whether there exist small physical parameters or not; the method provides a convenient way to guarantee the convergence of approximation series; and also the method provides great freedom to choose the equation type of linear subproblems and the base functions of solutions. As a result, the HAM overcomes the restrictions of all other analytic approximation methods mentioned above and is valid for highly nonlinear problems [33].

\section{Buckling of Elastic Columns with Continuous Restraints}

A uniform homogeneous column which is continuously restrained along its length with flexural rigidity $E I$ and length $L$ is investigated. The restraint consists of lateral springs of stiffness $k$ per unit length.

Governing equation for the buckling of an Euler column with continuous elastic restraints in Figure 1 is given by

$$
\frac{d^{2}}{d x^{2}}\left(E I \frac{d^{2} w}{d x^{2}}\right)+P \frac{d^{2} w}{d x^{2}}+k w=0
$$

If the governing equation (1) is divided by $E I$, then it is normalized by defining nondimensional displacement and length $\bar{w}=w / L, \bar{x}=x / L$, respectively. Then normalized governing equation becomes

$$
\frac{d^{4} \bar{w}}{d \bar{x}^{4}}+\alpha \frac{d^{2} \bar{w}}{d \bar{x}^{2}}+\beta w=0, \quad \text { where } \alpha=\frac{P L^{2}}{E I} \text { and } \beta=\frac{k L^{4}}{E I}
$$

Investigation of buckling loads for continuously restrained elastic columns with five different support cases will be conducted via HAM throughout the study.

The general solution of governing equation and stability criteria for the columns with different end conditions are given in Wang et al. [6]. These end conditions can be seen in Figure 2.

The stability criteria for the columns considered in this study [6] are given in Table 1 . 
TABLE 1: The stability criteria for continuously restrained elastic columns.

\begin{tabular}{lc}
\hline Column & Stability criteria \\
\hline$C-F$ column & {$\left[\alpha\left(S^{2}+T^{2}\right)-2 S^{2} T^{2}\right] \cos T \cos S-\alpha\left(S^{2}+T^{2}\right)+\left(S^{4}+T^{4}\right)+S T\left[2 \alpha-\left(S^{2}+T^{2}\right)\right] \sin T \sin S=0$} \\
$P-P$ column & $\sin T=0$ \\
$C-P$ column & $T \cos T \sin S-S \sin T \cos S=0$ \\
$C-C$ column & $2 S T[\cos T \cos S-1]+\left(S^{2}+T^{2}\right) \sin T \sin S=0$ \\
$C-S$ column & $T \sin T \cos S-S \cos T \sin S=0$ \\
\hline
\end{tabular}

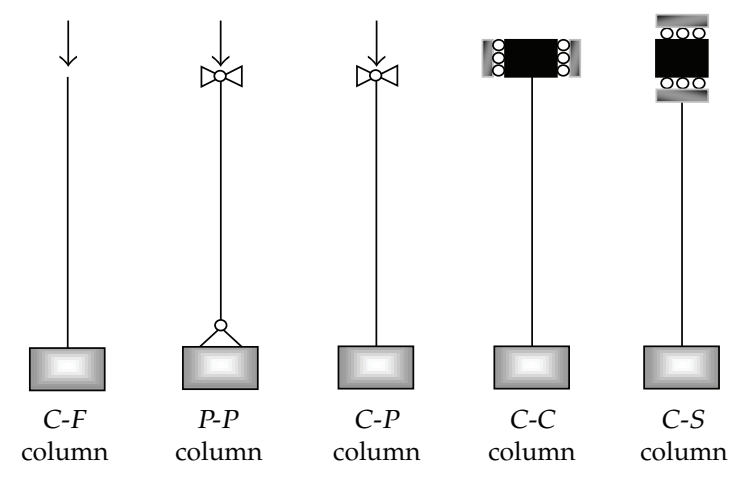

FIGURE 2: Various end conditions for restrained columns.

Where

$$
\begin{aligned}
& S=\sqrt{\frac{\alpha}{2}-\sqrt{\left(\frac{\alpha}{2}\right)^{2}-\beta}} \\
& T=\sqrt{\frac{\alpha}{2}+\sqrt{\left(\frac{\alpha}{2}\right)^{2}-\beta}}
\end{aligned}
$$

The classical boundary conditions [6] are as follows:

Clamped (Fixed) End: $w=0, \quad \frac{d w}{d x}=0$,

Pinned End: $\quad w=0, \quad \frac{d^{2} w}{d x^{2}}=0$,

Free End: $\quad \frac{d^{2} w}{d x^{2}}=0, \quad \frac{d^{3} w}{d x^{3}}+\alpha \frac{d w}{d x}=0$,

Sliding Restraint: $\quad \frac{d w}{d x}=0, \frac{d^{3} w}{d x^{3}}+\alpha \frac{d w}{d x}=0$.

\section{HAM Formulation of the Problem}

To solve (3) by means of homotopy analysis method, let us define $N[\phi(x ; q)]$ as follows:

$$
(1-q) L\left[\phi(x ; q)-w_{0}(x)\right]=q \hbar H(x) N[\phi(x ; q)],
$$

where $\phi(x ; q)$ is an unknown function to be determined and $N[\phi(x ; q)]$ is given by

$$
N[\phi(x ; q)]=\phi^{(v)}(x ; q)+\alpha \phi^{\prime \prime}(x ; q)+\beta \phi(x ; q) .
$$

The high-order deformation equation is as follows:

$$
\begin{aligned}
& w_{m}(x)= \chi_{m} w_{m-1}(x) \\
&+\hbar \int_{0}^{x} \int_{0}^{\tau} \int_{0}^{\zeta} \int_{0}^{\psi}\left[w_{m-1}^{(i v)}(\xi)+\alpha w_{m-1}^{\prime \prime}(\xi)\right. \\
&\left.+\beta w_{m-1}(\xi)\right] d \xi d \psi d \zeta d \tau,
\end{aligned}
$$

where

$$
\chi_{m}= \begin{cases}0, & m \leq 1 \\ 1, & m>1\end{cases}
$$

Starting with $w_{0}(x)$, successively $w_{i}(x), i=1,2,3, \ldots$ are determined by the so-called high-order deformation equation (8); then the solution is

$$
w(x)=w_{0}(x)+\sum_{m=1}^{\infty} w_{m}(x)
$$

\section{Critical Buckling Loads for Continuously Restrained Elastic Columns}

A cubic polynomial $w_{0}(x)=a x^{3}+b x^{2}+c x+d$ is chosen as an initial approximation due to four boundary conditions for each case. This polynomial has been successfully used in previous studies employing different analytical approximate techniques. This polynomial has also been used as the interpolation function in the finite element analysis of Euler beams or columns. Hence, the initial approximation is expected to produce good results. The approximation includes four unknown coefficients which will be found by substituting four boundary conditions into the solution. We successively 
obtain $w_{i}(x), i=1,2,3, \ldots$ by the $m$ th-order deformation equation (3):

$$
\begin{aligned}
w_{1}(x)= & \frac{1}{12} b \hbar \alpha x^{4}+\frac{1}{20} a \hbar \alpha x^{5}+\frac{1}{24} d \hbar \beta x^{4}+\frac{1}{120} c \hbar \beta x^{5} \\
& +\frac{1}{360} b \hbar \beta x^{6}+\frac{1}{840} a \hbar \beta x^{7}, \\
w_{2}(x)= & \frac{1}{12} b \hbar \alpha x^{4}+\frac{1}{12} b \hbar^{2} \alpha x^{4}+\frac{1}{20} a \hbar \alpha x^{5}+\frac{1}{20} a \hbar^{2} \alpha x^{5} \\
& +\frac{1}{360} b \hbar^{2} \alpha^{2} x^{6}+\frac{1}{840} a \hbar^{2} \alpha^{2} x^{7}+\frac{1}{24} d \hbar \beta x^{4} \\
& +\frac{1}{24} d \hbar^{2} \beta x^{4}+\frac{1}{120} c \hbar \beta x^{5}+\frac{1}{120} c \hbar^{2} \beta x^{5} \\
& +\frac{1}{360} b \hbar \beta x^{6}+\frac{1}{360} b \hbar^{2} \beta x^{6}+\frac{1}{840} a \hbar \beta x^{7} \\
& +\frac{1}{840} a \hbar^{2} \beta x^{7}+\frac{1}{720} d \hbar^{2} \alpha \beta x^{6}+\frac{1}{5040} c \hbar^{2} \alpha \beta x^{7} \\
& +\frac{1}{10080} b \hbar^{2} \alpha \beta x^{8}+\frac{1}{30240} a \hbar^{2} \alpha \beta x^{9} \\
& +\frac{1}{40320} d \hbar^{2} \beta^{2} x^{8}+\frac{1}{362880} c \hbar^{2} \beta^{2} x^{9} \\
& +\frac{1}{1814400} b \hbar^{2} \beta^{2} x^{10}+\frac{1}{6652800} a \hbar^{2} \beta^{2} x^{11},
\end{aligned}
$$

Ten iterations are conducted. In this way, we get the final approximation as follows:

$$
\begin{aligned}
W_{10}(x, \hbar) & =\sum_{n=0}^{10} w_{n}(x) \\
& =w_{0}(x)+w_{1}(x)+w_{2}(x)+\cdots+w_{10}(x) .
\end{aligned}
$$

By substituting (12) into the boundary conditions, we obtained four homogeneous equations. By representing these equations in the matrix form by coefficient matrix $[C(\alpha, \beta)]$, we obtained the following equation in matrix form:

$$
[C(\alpha, \beta)]\left[\begin{array}{l}
a \\
b \\
c \\
d
\end{array}\right]=\left[\begin{array}{l}
0 \\
0 \\
0 \\
0
\end{array}\right],
$$

where $a, b, c$, and $d$ are the unknown constants, which has been introduced in the initial approximation. For a nontrivial solution the determinant of the coefficient matrix $[C(\alpha, \beta)]$ must vanish. Then, the problem takes the following form:

$$
\operatorname{Det}[C(\alpha, \beta)]=0 \text {. }
$$

The smallest positive real root of (14) is the normalized critical buckling load. The next positive real root is the normalized buckling load for second mode, and so on. Equation (14)

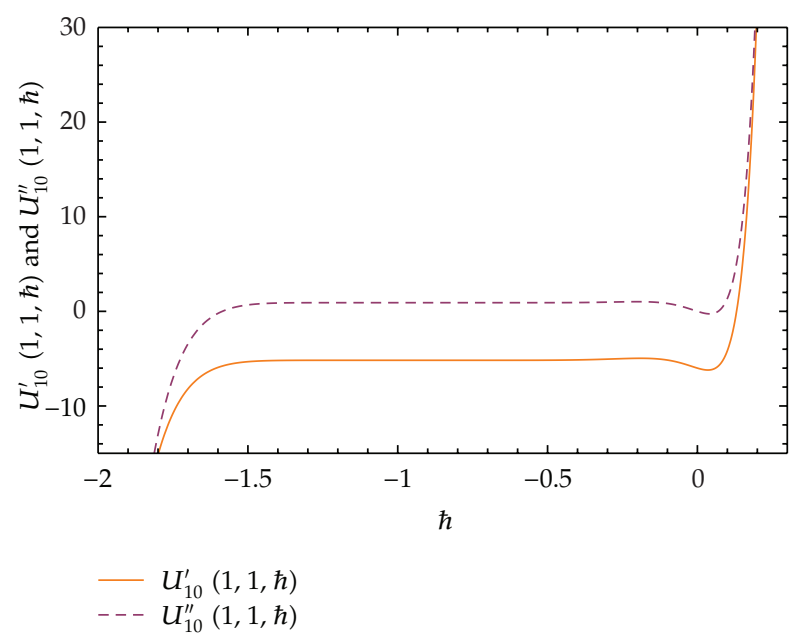

FIgURE 3: The $\hbar$ curves of $U_{10}^{\prime}(1,1, \hbar)$ and $U_{10}^{\prime \prime}(1,1, \hbar)$ for $C-S$ column.

depends on the stability parameter $\alpha$, the restraint stiffness parameter $\beta$, and the convergence control parameter $\hbar$. Then we define the function $U(\alpha, \beta, \hbar)$ as follows:

$$
U(\alpha, \beta, \hbar)=\operatorname{Det}[C(\alpha, \beta)] .
$$

And, then we plot the $\hbar$ curves of the $U_{10}^{\prime}(\alpha, \beta, \hbar)$ and $U_{10}^{\prime \prime}(\alpha, \beta, \hbar)$ in order to find convergence region of the $\hbar$, where prime denotes derivatives of $U(\alpha, \beta, \hbar)$ with respect to $\alpha$.

4.1. Clamped-Free (C-F) Column. Substituting the 10th-order approximation $W_{10}(\alpha, \beta, \hbar)$ into the boundary conditions of $C-F$ column, we get coefficient matrix $\left[C_{C-F}(\alpha, \beta)\right]$. We define the function $U(\alpha, \beta, \hbar)$ as follows:

$$
U(\alpha, \beta, \hbar)=\operatorname{Det}\left[C_{C-F}(\alpha, \beta)\right] .
$$

Then, the $\hbar$ curves of $U_{10}^{\prime}(1,1, \hbar)$ and $U_{10}^{\prime \prime}(1,1, \hbar)$ are obtained in Figure 3 , and the valid region of $\hbar$ is approximated as $-1,5<\hbar<-0,3$.

Finally the critical buckling load obtained from (14) for $\hbar=-0,99$ is 2,4674 .

4.2. Pinned-Pinned (P-P) Column. Substituting the 10thorder approximation $W_{10}(\alpha, \beta, \hbar)$ into the boundary conditions of $P-P$ column, we get coefficient matrix $\left[C_{P-P}(\alpha, \beta)\right]$. We define the function $U(\alpha, \beta, \hbar)$ as follows:

$$
U(\alpha, \beta, \hbar)=\operatorname{Det}\left[C_{P-P}(\alpha, \beta)\right] .
$$

Then the $\hbar$ curves of $U_{10}^{\prime}(1,1, \hbar)$ and $U_{10}^{\prime \prime}(1,1, \hbar)$ are obtained in Figure 4 , and the valid region of $\hbar$ is about $-1,6<\hbar<$ $-0,4$.

Finally, the critical buckling load obtained from (14) for $\hbar=-0,995$ is 9,8696 . 


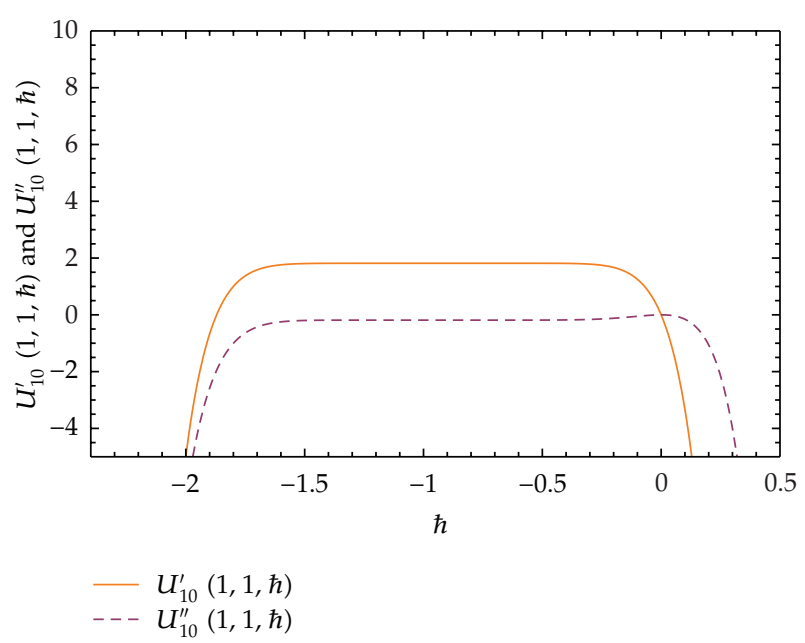

FIgure 4: The $\hbar$ curves of $U_{10}^{\prime}(1,1, \hbar)$ and $U_{10}^{\prime \prime}(1,1, \hbar)$ for $P$-P column.

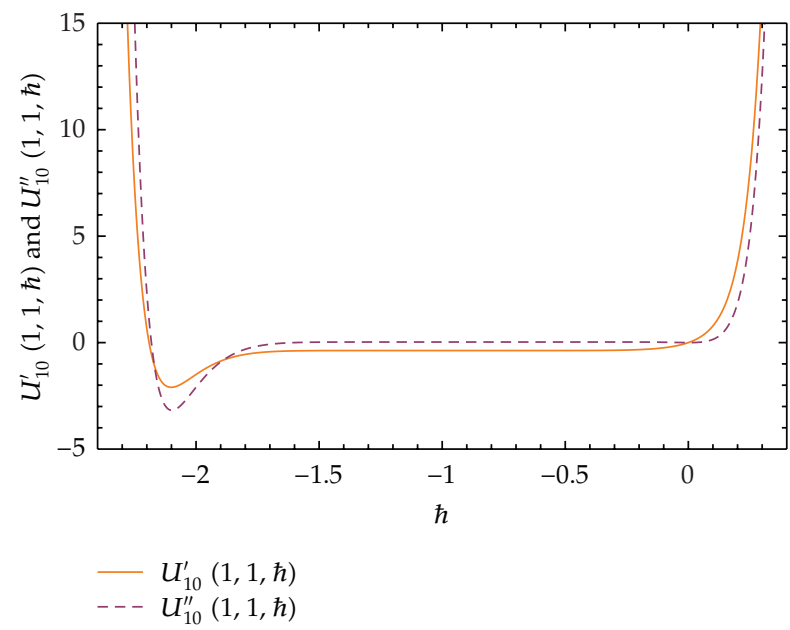

Figure 5: The $\hbar$ curves of $U_{10}^{\prime}(1,1, \hbar)$ and $U_{10}^{\prime \prime}(1,1, \hbar)$ for $C$-P column.

4.3. Clamped-Pinned (C-P) Column. Substituting the 10th order approximation $W_{10}(\alpha, \beta, \hbar)$ into the boundary conditions of $C-P$ column, we get coefficient matrix $\left[C_{C-P}(\alpha, \beta)\right]$. We define the function $U(\alpha, \beta, \hbar)$ as follows:

$$
U(\alpha, \beta, \hbar)=\operatorname{Det}\left[C_{C-P}(\alpha, \beta)\right] .
$$

Then the $\hbar$ curves of $U_{10}^{\prime}(1,1, \hbar)$ and $U_{10}^{\prime \prime}(1,1, \hbar)$ are obtained in Figure 5, and the valid region of $\hbar$ is as follows: $-1,75<$ $\hbar<-0,3$.

Finally the critical buckling load obtained from (14) for $\hbar=-0,97$ is 20,1907 .

4.4. Clamped-Clamped (C-C) Column. Substituting the 10thorder approximation $W_{10}(\alpha, \beta, \hbar)$ into the boundary conditions of $C$ - $C$ column, we get coefficient matrix $\left[C_{C-C}(\alpha, \beta)\right]$. We define the function $U(\alpha, \beta, \hbar)$ as follows:

$$
U(\alpha, \beta, \hbar)=\operatorname{Det}\left[C_{C-C}(\alpha, \beta)\right] .
$$

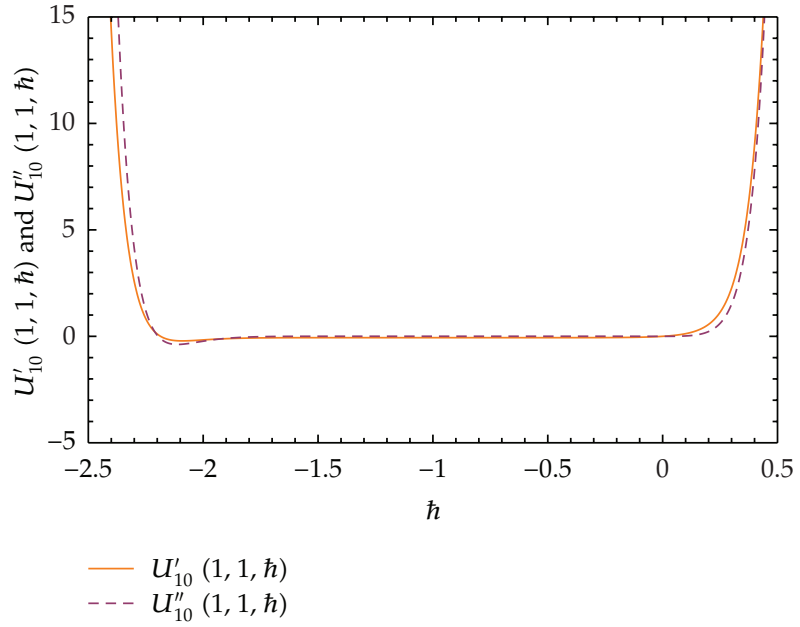

Figure 6: The $\hbar$ curves of $U_{10}^{\prime}(1,1, \hbar)$ and $U_{10}^{\prime \prime}(1,1, \hbar)$ for $C$-C column.

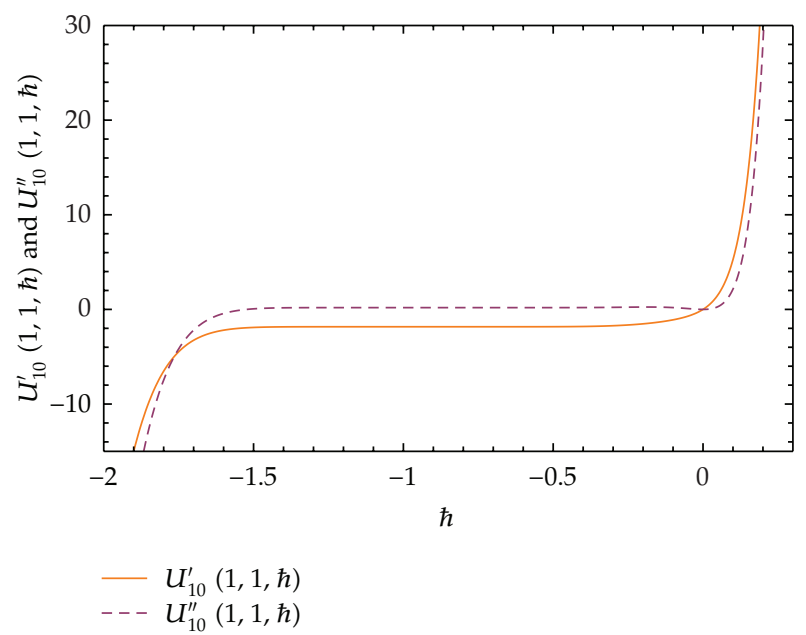

Figure 7: The $\hbar$ curves of $U_{10}^{\prime}(1,1, \hbar)$ and $U_{10}^{\prime \prime}(1,1, \hbar)$ for $C-S$ column.

Then the $\hbar$ curves of $U_{10}^{\prime}(1,1, \hbar)$ and $U_{10}^{\prime \prime}(1,1, \hbar)$ are obtained in Figure 6 , and the valid region of $\hbar$ can be $-1,8<\hbar<-0,1$.

Finally the critical buckling load obtained from (14) for $\hbar=-0,96868$ is 39,4784 .

4.5. Clamped-Sliding Restraint (C-S) Column. Substituting the 10th-order approximation $W_{10}(\alpha, \beta, \hbar)$ into the boundary conditions of $C-S$ column, we get coefficient matrix $\left[C_{C-S}(\alpha, \beta)\right]$. We define the function $U(\alpha, \beta, \hbar)$ as follows:

$$
U(\alpha, \beta, \hbar)=\operatorname{Det}\left[C_{C-S}(\alpha, \beta)\right] \text {. }
$$

Then, the $\hbar$ curves of $U_{10}^{\prime}(1,1, \hbar)$ and $U_{10}^{\prime \prime}(1,1, \hbar)$ are obtained in Figure 7, and the valid region of $\hbar$ can be $-1,55<\hbar<$ $-0,37$.

Finally the critical buckling load obtained from (14) for $\hbar=-0,9909$ is 9,8696 .

The exact solutions for the presented cases are obtained via stability criteria provided by Wang et al. [6]. In 
TABLE 2: Comparison of exact and HAM solutions of the buckling loads for $\beta=0$ with ten iterations.

\begin{tabular}{lccccccccrcr}
\hline \multirow{2}{*}{ Mode } & \multicolumn{2}{c}{$C-F$ column } & \multicolumn{2}{c}{$P-P$ column } & \multicolumn{2}{c}{$C-P$ column } & \multicolumn{2}{c}{$C-C$ column } & \multicolumn{2}{c}{$C-S$ column } \\
& Exact & HAM & Exact & HAM & Exact & HAM & Exact & HAM & Exact & HAM \\
\hline 1 & 2.4674 & 2.4674 & 9.8696 & 9.8696 & 20.1907 & 20.1907 & 39.4784 & 39.4784 & 9.8696 & 9.8696 \\
2 & 22.2066 & 22.2066 & 39.4784 & 39.4779 & 59.6795 & 59.6813 & 80.7629 & 80.9336 & 39.4784 & 39.4784 \\
\hline
\end{tabular}

TABLE 3: Comparison of exact and HAM solutions of the buckling loads for $\beta=50$ with ten iterations.

\begin{tabular}{lcccccccccc}
\hline \multirow{2}{*}{ Mode } & \multicolumn{2}{c}{$C-F$ Column } & \multicolumn{2}{c}{$P$-P Column } & \multicolumn{2}{c}{$C-P$ Column } & \multicolumn{2}{c}{$C$-C Column } & \multicolumn{2}{c}{$C-S$ Column } \\
& Exact & HAM & Exact & HAM & Exact & HAM & Exact & HAM & Exact & HAM \\
\hline 1 & 8.8614 & 8.8614 & 14.9357 & 14.9357 & 24.2852 & 24.2852 & 43.2606 & 43.2606 & 23.5717 & 23.5717 \\
2 & 33.0879 & 33.0873 & 40.7449 & 40.7442 & 61.0966 & 61.1003 & 81.7943 & 82.0059 & 44.4104 & 44.4053 \\
\hline
\end{tabular}

TABLE 4: Comparison of exact and HAM solutions of the buckling loads for $\beta=100$ with ten iterations.

\begin{tabular}{lcccccccccc}
\hline \multirow{2}{*}{ Mode } & \multicolumn{2}{c}{$C-F$ column } & \multicolumn{2}{c}{$P-P$ column } & \multicolumn{2}{c}{$C-P$ column } & \multicolumn{2}{c}{$C-C$ column } & \multicolumn{2}{c}{$C-S$ column } \\
& Exact & HAM & Exact & HAM & Exact & HAM & Exact & HAM & Exact & HAM \\
\hline 1 & 11.9964 & 11.9964 & 20.0017 & 20.0017 & 28.3066 & 28.3066 & 47.0066 & 47.0066 & 32.6690 & 32.6690 \\
2 & 45.2659 & 45.2574 & 42.0114 & 42.0105 & 62.5613 & 62.5678 & 82.8246 & 83.0859 & 52.8965 & 52.8105 \\
\hline
\end{tabular}

TABLE 5: Absolute errors of HAM approximations for the first buckling mode.

\begin{tabular}{lccccc}
\hline & $C-F$ column & $P-P$ column & $C-P$ column & $C-C$ column & $C-S$ column \\
\hline$\beta=0$ & $1.30562 E-13$ & $1.03727 E-12$ & 0.00000 & 0.00000 & $5.39109 E-11$ \\
$\beta=50$ & $9.81515 E-10$ & $1.26009 E-10$ & $1.09165 E-7$ & $4.64191 E-5$ & $1.50249 E-7$ \\
$\beta=100$ & $3.04503 E-8$ & $1.65444 E-8$ & $7.37428 E-7$ & $5.9300 E-7$ & $1.81936 E-5$ \\
\hline
\end{tabular}

TABLE 6: Percent relative errors of HAM approximations for the second buckling mode.

\begin{tabular}{lccccc}
\hline & $C-F$ column & $P-P$ column & $C-P$ column & $C$-C column & $C-S$ column \\
\hline$\beta=0$ & 0.00000621 & 0.00124745 & 0.00303663 & 0.21128177 & 0.00008617 \\
$\beta=50$ & 0.00163762 & 0.00173402 & 0.00599296 & 0.25870613 & 0.01135007 \\
$\beta=100$ & 0.01874692 & 0.00227055 & 0.01038389 & 0.31551889 & 0.16247717 \\
\hline
\end{tabular}

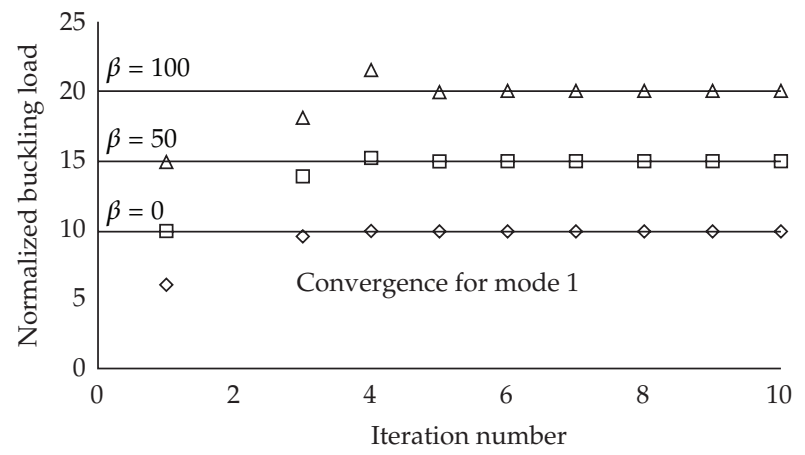

FIGURE 8: Convergence of solutions through the iterations for the first mode of $P-P$ column.

the following tables, the buckling loads for the first two modes are given and compared with exact results.

From Tables 2-4 it is observed that normalized buckling loads for the first mode which is the critical buckling load are in excellent agreement with analytical results. Absolute errors for these cases are given in Table 5.
The differences in the results of the second mode exist due to lack of iterations provided by the method. Additional iterations would improve the results for the second mode. However, the results for the second mode are still in good agreement with the exact results. Relative errors for this case are provided in the following Table 6.

These percent relative errors show that the presented solution is in good agreement with analytical ones. Convergence of solutions for the PP column is simulated in Figures 8 and 9. Solid lines show the exact solutions for different normalized spring stiffnesses.

Same convergence behaviors are observed for all cases considered in this study. Furthermore, Figure 8 shows that at least 6 iterations are required for the first mode, and Figure 9 shows that at least 10 iterations are required for the second mode to obtain satisfactory results from the analysis.

\section{Conclusions}

In this work, a reliable algorithm based on the HAM to obtain the normalized buckling loads of the Euler columns 


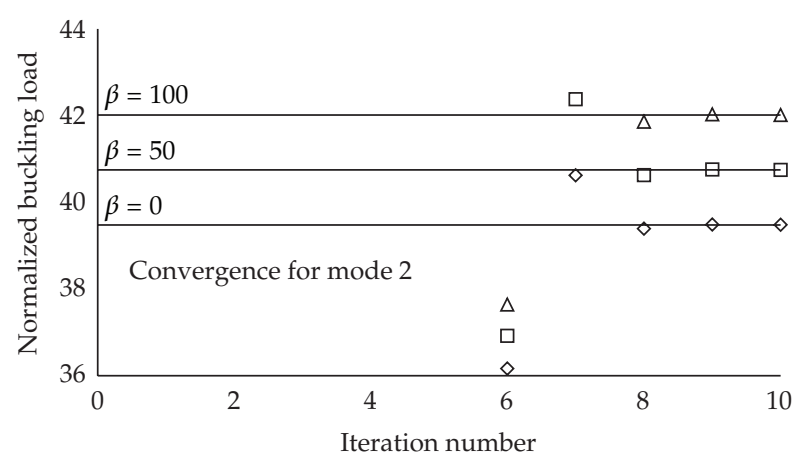

FIGURE 9: Convergence of solutions through the iterations for the second mode of $P-P$ column.

with constant flexural stiffness is presented. Several cases are given to illustrate the validity and accuracy of this procedure. The series solutions of (3) by HAM contain the auxiliary parameter $\hbar$. In general, by means of the so-called $\hbar$-curve, it is straightforward to choose a proper value of $\hbar$ which ensures that the series solution is convergent. Figures 2, 3, 4,5 , and 6 show the $\hbar$-curves obtained from the $m$ th-order HAM approximation solutions. From these figures, the valid regions of $\hbar$ correspond to the line segments nearly parallel to the horizontal axis. By the use of the proper value with this parameter, two buckling loads for the first and second modes are obtained. The method will provide the results for the following modes if additional iterations are introduced in the analysis. The buckling loads are positive real roots of the characteristic equations which are obtained consecutively. This is a huge advantage, because it is still very difficult to obtain those roots consecutively even with a mathematics software. As a result, HAM is an efficient, powerful, and accurate tool for determining the buckling loads of Euler columns.

\section{References}

[1] L. Euler, "Die altitudine colomnarum sub proprio pondere corruentium," Acta Academiae Scientiarum Imperialis Petropolitanae, 1778, in Latin.

[2] A. G. Greenhill, "Determination of the greatest height consistent with stability that a vertical pole on mast can be made and of the greatest height to which a tree of given proportions can grow," in Proceedings of the Cambridge Philosophical Society IV, 1883.

[3] A. N. Dinnik, "Design of columns of varying cross-section," Transactions of the ASME, vol. 51, 1929.

[4] T. R. Karman and M. A. Biot, Mathematical Methods in Engineering, McGraw Hill, New York, NY, USA, 1940.

[5] S. P. Timoshenko and J. M. Gere, Theory of Elastic Stability, McGraw Hill, New York, NY, USA, 1961.

[6] C. M. Wang, C. Y. Wang, and J. N. Reddy, Exact Solutions for Buckling of Structural Members, CRC Press LLC, Florida, Fla, USA, 2005.

[7] H. Bleich, Buckling Strength of Metal Structures, McGraw-Hill, New York, NY, USA, 1952.

[8] A. N. Dinnik, "Design of columns of varying cross-section," Transactions of the ASME, Applied Mechanics, vol. 51, 1932.
[9] I. Elishakoff and F. Pellegrini, "Exact and effective approximate solution solutions of some divergent type non-conservative problems," Journal of Sound and Vibration, vol. 114, pp. 144-148, 1987.

[10] I. Elishakoff and F. Pellegrini, "Application of bessel and lommel functions, and the undetermined multiplier galerkin method version, for instability of non-uniform column," Journal of Sound and Vibration, vol. 115, no. 1, pp. 182-186, 1987.

[11] I. Elishakoff and F. Pellegrini, "Exact solutions for buckling of some divergence-type nonconservative systems in terms of bessel and lommel functions," Computer Methods in Applied Mechanics and Engineering, vol. 66, no. 1, pp. 107-119, 1988.

[12] M. Eisenberger, "Buckling loads for variable cross-section members with variable axial forces," International Journal of Solids and Structures, vol. 27, no. 2, pp. 135-143, 1991.

[13] J. M. Gere and W. O. Carter, "Critical buckling loads for tapered columns," Journal of Structural Engineering ASCE, vol. 88, no. 1, pp. 1-11, 1962.

[14] F. Arbabi and F. Li, "Buckling of variable cross-section columns integral equation approach," Journal of Structural Engineering ASCE, vol. 117, no. 8, 1991.

[15] A. Siginer, "Buckling of columns of variable flexural rigidity," Journal of Engineering Mechanics, ASCE, vol. 118, no. 3, pp. 543640, 1992.

[16] Q. Li, H. Cao, and G. Li, "Stability analysis of a bar with multisegments of varying cross-section," Computers and Structures, vol. 53, no. 5, pp. 1085-1089, 1994.

[17] L. Qiusheng, C. Hong, and L. Guiqing, "Stability analysis of bars with varying cross-section," International Journal of Solids and Structures, vol. 32, no. 21, pp. 3217-3228, 1995.

[18] Q. Li, H. Cao, and G. Li, "Static and dynamic analysis of straight bars with variable cross-section," Computers and Structures, vol. 59, no. 6, pp. 1185-1191, 1996.

[19] J. H. B. Sampaio and J. R. Hundhausen, "A mathematical model and analytical solution for buckling of inclined beam-columns," Applied Mathematical Modelling, vol. 22, no. 6, pp. 405-421, 1998.

[20] J. B. Keller, “The shape of the strongest column," Archive for Rational Mechanics and Analysis, vol. 5, no. 1, pp. 275-285, 1960.

[21] I. Tadjbakhsh and J. B. Keller, "Strongest columns and isoperimetric inequalities for eigenvalues," Journal of Applied Mechanics ASME, vol. 29, pp. 159-164, 1962.

[22] J. E. Taylor, “The strongest column-an energy approach," Journal of Applied Mechanics ASME, vol. 34, pp. 486-487, 1967.

[23] M. T. Atay and S. B. Coşkun, "Elastic stability of Euler columns with a continuous elastic restraint using variational iteration method," Computers and Mathematics with Applications, vol. 58, no. 11-12, pp. 2528-2534, 2009.

[24] S. B. Coşkun and M. T. Atay, "Determination of critical buckling load for elastic columns of constant and variable cross-sections using variational iteration method," Computers and Mathematics with Applications, vol. 58, no. 11-12, pp. 2260-2266, 2009.

[25] M. T. Atay, "Determination of buckling loads of tilted buckled column with varying flexural rigidity using variational iteration method," International Journal of Nonlinear Sciences and Numerical Simulation, vol. 11, no. 2, pp. 97-103, 2010.

[26] F. Okay, M. T. Atay, and S. B. Coçkun, "Determination of buckling loads and mode shapes of a heavy vertical column under its own weight using the variational iteration method," International Journal of Nonlinear Sciences and Numerical Simulation, vol. 11, no. 10, pp. 851-857, 2010. 
[27] S. B. Coşkun, "Determination of critical buckling loads for euler columns of variable flexural stiffness with a continuous elastic restraint using homotopy perturbation method," International Journal of Nonlinear Sciences and Numerical Simulation, vol. 10, no. 2, pp. 191-197, 2009.

[28] M. T. Atay, "Determination of critical buckling loads for variable stiffness euler columns using homotopy perturbation method," International Journal of Nonlinear Sciences and Numerical Simulation, vol. 10, no. 2, pp. 199-206, 2009.

[29] S. B. Coşkun, "Analysis of tilt-buckling of euler columns with varying flexural stiffness using homotopy perturbation method," Mathematical Modelling and Analysis, vol. 15, no. 3, pp. 275286, 2010.

[30] S. Pinarbasi, "Stability analysis of non-uniform rectangular beams using homotopy perturbation method," Mathematical Problems in Engineering, vol. 2012, Article ID 197483, 18 pages, 2012.

[31] Y. Huang and Q. Z. Luo, "A simple method to determine the critical buckling loads for axially inhomogeneous beams with elastic restraint," Computers and Mathematics with Applications, vol. 61, no. 9, pp. 2510-2517, 2011.

[32] Z.X. Yuan and X. W. Wang, "Buckling and post-buckling analysis of extensible beam-columns by using the differential quadrature method," Computers \& Mathematics with Applications, vol. 62, no. 12, pp. 4499-4513, 2011.

[33] S. J. Liao, Beyond Perturbation: Introduction to the Homotopy Analysis Method, CRC Press, 2004.

[34] S. J. Liao, "Homotopy analysis method: a new analytic method for nonlinear problems," Applied Mathematics and Mechanics, vol. 19, no. 10, pp. 957-962, 1998.

[35] S. J. Liao, "A uniformly valid analytic solution of two-dimensional viscous flow over a semi-infinite flat plate," Journal of Fluid Mechanics, vol. 385, pp. 101-128, 1999.

[36] S. J. Liao, "An explicit analytic solution to the Thomas-Fermi equation," Applied Mathematics and Computation, vol. 144, no. 2-3, pp. 495-506, 2003.

[37] T. Hayat, T. Javed, and M. Sajid, "Analytic solution for rotating flow and heat transfer analysis of a third-grade fluid," Acta Mechanica, vol. 191, no. 3-4, pp. 219-229, 2007.

[38] T. Hayat, S. B. Khan, M. Sajid, and S. Asghar, "Rotating flow of a third grade fluid in a porous space with Hall current," Nonlinear Dynamics, vol. 49, no. 1-2, pp. 83-91, 2007.

[39] S. Abbasbandy, "Homotopy analysis method for heat radiation equations," International Communications in Heat and Mass Transfer, vol. 34, no. 3, pp. 380-387, 2007.

[40] S. Abbasbandy and E. Shivanian, "A new analytical technique to solve Fredholm's integral equations," Numerical Algorithms, vol. 56, no. 1, pp. 27-43, 2011.

[41] S. Abbasbandy and F. S. Zakaria, "Soliton solutions for the fifthorder KdV equation with the homotopy analysis method," Nonlinear Dynamics, vol. 51, no. 1-2, pp. 83-87, 2008.

[42] Z. Wang, L. Zou, and H. Zhang, "Applying homotopy analysis method for solving differential-difference equation," Physics Letters A, vol. 369, no. 1-2, pp. 77-84, 2007.

[43] A. S. Bataineh, M. S. M. Noorani, and I. Hashim, "Solutions of time-dependent Emden-Fowler type equations by homotopy analysis method," Physics Letters A, vol. 371, no. 1-2, pp. 72-82, 2007.

[44] M. Inc, "On exact solution of Laplace equation with Dirichlet and Neumann boundary conditions by the homotopy analysis method," Physics Letters A, vol. 365, no. 5-6, pp. 412-415, 2007.
[45] F. Awawdeh, A. Adawi, and S. Al-Shara, "Analytic solution of multi pantograph equation," Journal of Applied Mathematics and Decision Sciences, vol. 2008, Article ID 605064, 11 pages, 2008. 


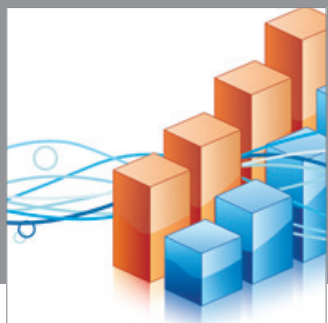

Advances in

Operations Research

mansans

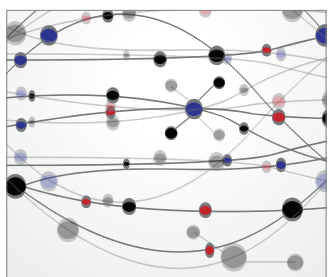

The Scientific World Journal
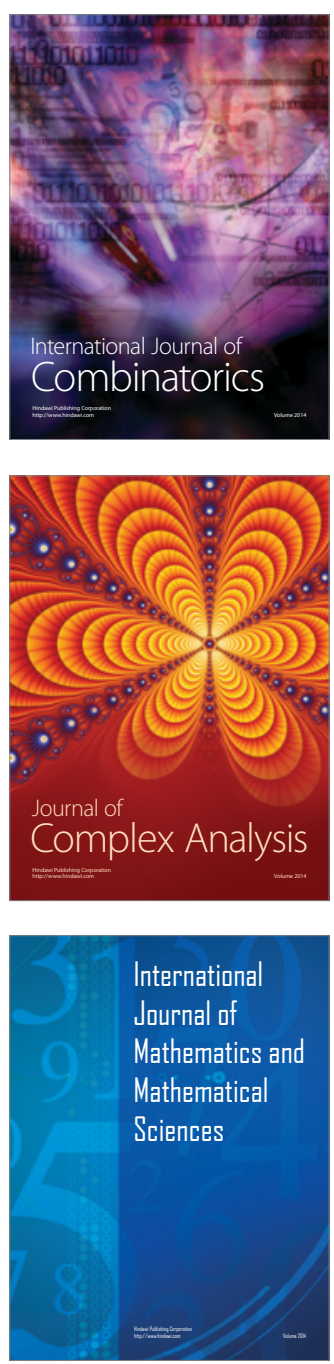
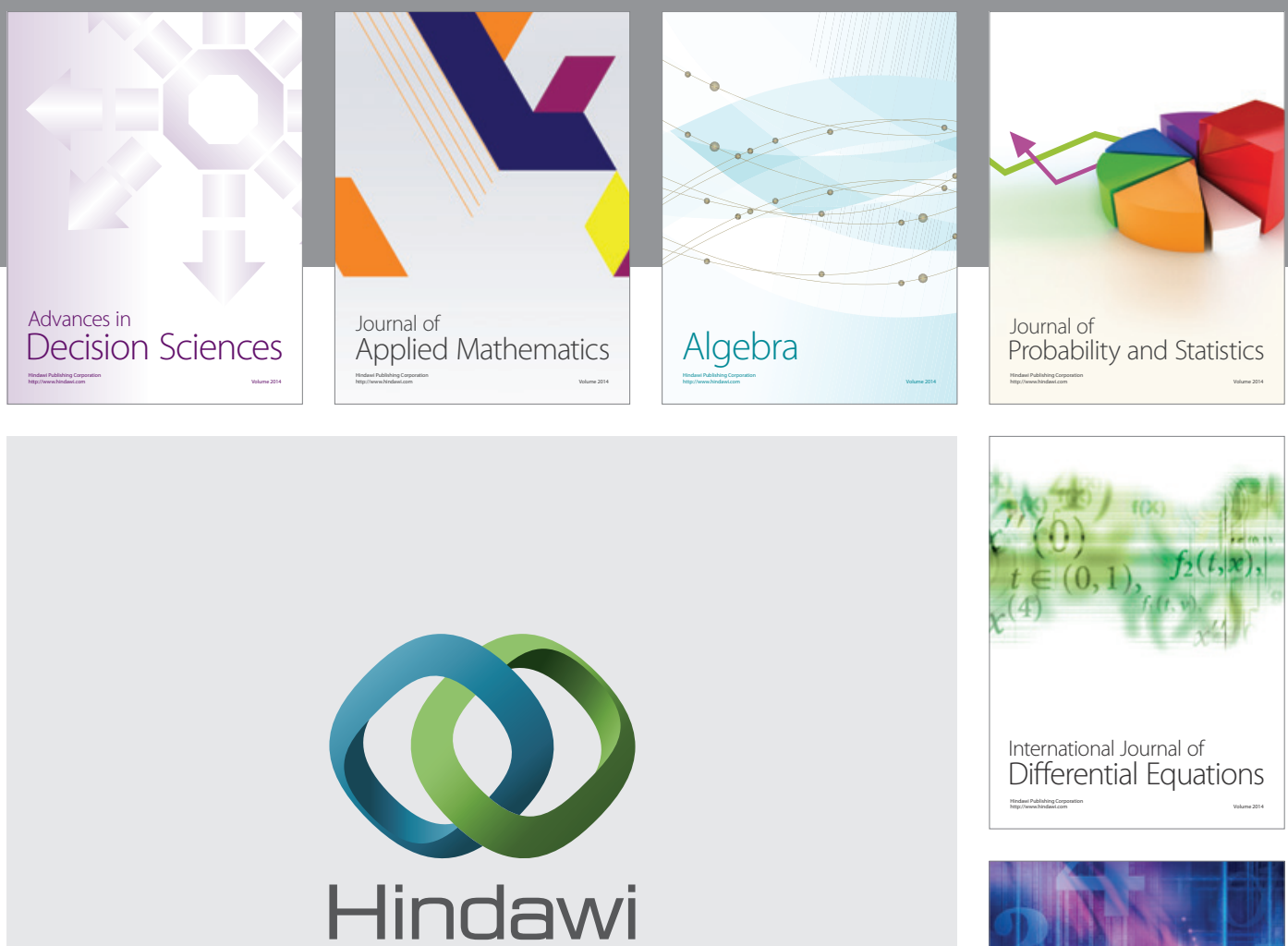

Submit your manuscripts at http://www.hindawi.com
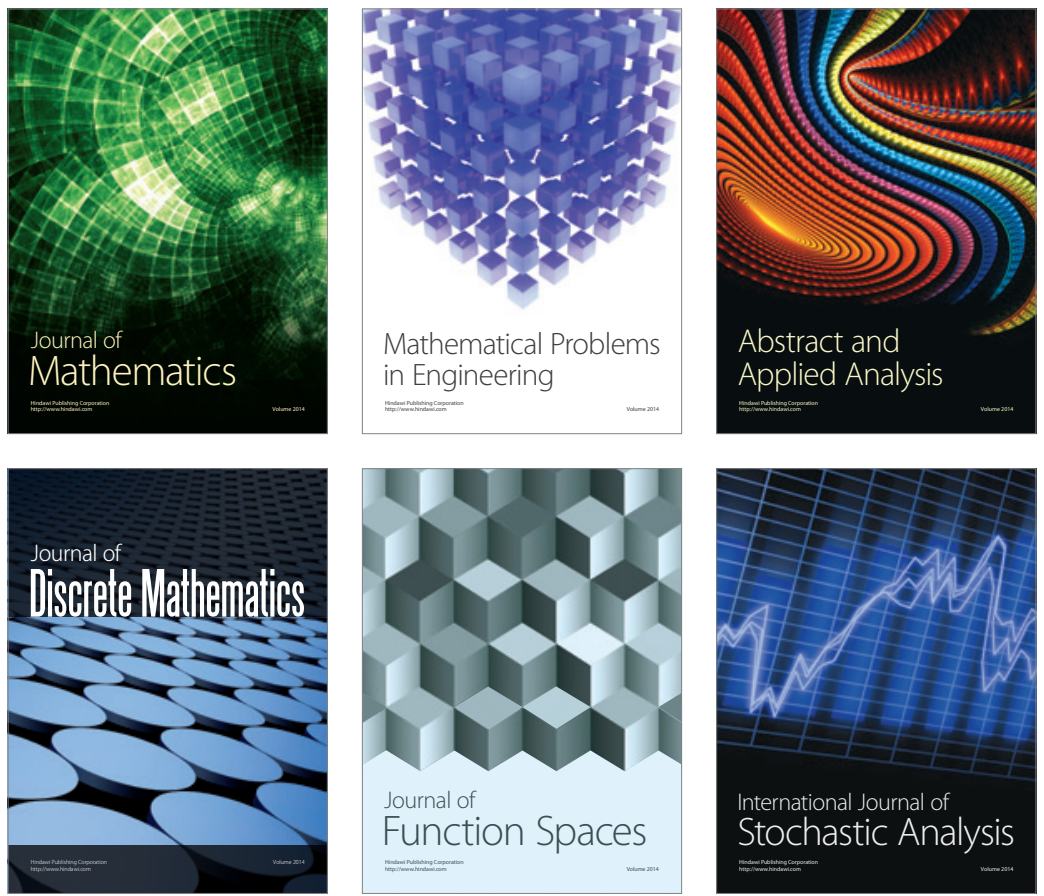

Journal of

Function Spaces

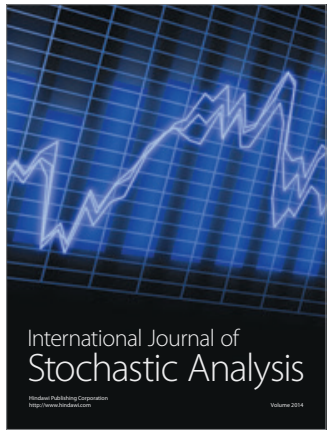

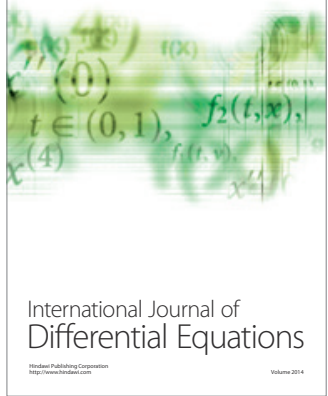
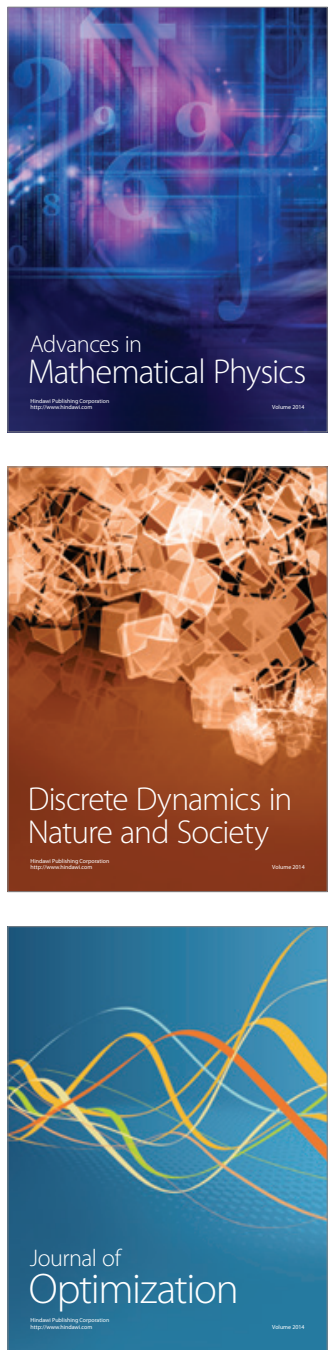